EPJ Web of Conferences 81, 02008 (2014)

DOI: $10.1051 /$ epjconf/ 20148102008

(C) Owned by the authors, published by EDP Sciences, 2014

\title{
The Muon Scattering Experiment (MUSE) at PSI and the proton radius puzzle
}

\author{
Michael Kohl ${ }^{1,2, a}$ for the MUSE Collaboration \\ ${ }^{1}$ Hampton University, Hampton, VA 23668, USA \\ 2 Jefferson Laboratory, Newport News, VA 23606, USA
}

\begin{abstract}
The unexplained large discrepancy of the proton charge radius measurements with muonic hydrogen Lamb shift and determinations from elastic electron scattering and Lamb shift in regular hydrogen of seven standard deviations is known as the proton radius puzzle. Suggested solutions of the puzzle range from possible errors in the experiments through unexpectedly large hadronic physics effects to new physics beyond the Standard Model. A new approach to verify the radius discrepancy in a systematic manner will be pursued with the Muon Scattering Experiment (MUSE) at PSI. The experiment aims to compare elastic cross sections, the proton elastic form factors, and the extracted proton charge radius with scattering of electrons and muons of either charge and under identical conditions. The difference in the observed radius will be probed with a high precision to verify the discrepancy. An overview of the experiment and the current status will be presented.
\end{abstract}

\section{Introduction}

The proton charge radius is an important quantity characterizing the proton charge distribution associated with the internal structure of the proton. The root-mean-square (rms) radius of the proton, $r_{p}$, is defined as the square root of the integral of the proton's charge density in the rest frame weighted with $r^{2}$, from the center to infinity. In relativistic Quantum Mechanics, the rms radius is equivalent to the first derivative of the proton charge form factor versus four-momentum transfer squared, $Q^{2}$, in the static limit of $Q^{2} \rightarrow 0$. Traditionally, the proton rms radius has been measured with elastic electron-proton scattering since the 1950's by investigating how the form factor changes near $Q^{2}=0$. Hofstadter et al. already got it right to a level of uncertainty that today marks the size of the proton radius puzzle [1]. Over the years, the proton charge radius from electron scattering experiments has stabilized around $0.87-0.88 \mathrm{fm}$ with an average of $r_{p}=(0.877 \pm 0.006)$ fm from the two latest published values from electron scattering $[2,3]$. The proton $\mathrm{rms}$ charge radius also contributes to the Lamb shift of the $2 \mathrm{~S}-2 \mathrm{P}$ atomic transitions of the hydrogen atom, due to the overlap of the atomic shells with the finite-sized nuclear center. The proton charge radius has been extracted from the Lamb shift of 2S-2P transitions in regular hydrogen in the framework of bound-state Quantum Electrodynamics (QED). The CODATA2010 value [7] of $r_{p}=(0.8775 \pm 0.0051) \mathrm{fm}$ is based on electron scattering and Lamb shift measurements from regular hydrogen. Both the Lamb shift and

\footnotetext{
a e-mail: kohlm@jlab.org
} 
electron scattering values have similar precision and are consistent with each other. More recently it became also feasible to use muonic hydrogen for Lamb shift measurements. Here the finite-size effect on the Lamb shift is strongly enhanced compared to regular hydrogen due to the higher mass of the muon and the tighter orbits of its atomic states. The resulting radius measurement is an order of magnitude more precise. In the first published paper [4], the proton charge radius was determined as $r_{p}=(0.84184 \pm 0.00067) \mathrm{fm}$, deviating from the CODATA2006 value [5] by five standard deviations. A follow-up work [6] evaluated additional transitions between substates of the hyperfine multiplets and found $r_{p}=(0.84087 \pm 0.00039) \mathrm{fm}$, consistent with the first value and with errors reduced even further. The discrepancy with the meanwhile more precise CODATA2010 [7] value has increased to over seven standard deviations, as much as $4 \%$ of the value. This unsatisfactory situation is known as the proton radius puzzle, which has been discussed in detail in a recent review [8]. Standard theory has been unable to explain this discrepancy in given frameworks. The biggest possible effects due to conventional physics are related to the muon vacuum polarization [9], which have been questioned [10], and due to the proton polarizability contribution to the effect of two-photon exchange, which could be different in size for $e p$ and $\mu p$ interaction [11-15]. It is controversial whether the size of the proton polarizability effect can be large enough to explain the radius discrepancy. The puzzle may be hidden in the bound state physics alone, such as in the size of the Rydberg constant, which is on the other hand known as one of the best measured quantities in physics. Finally it may be an effect that generally distinguishes electrons and muons. Such new physics scenarios have been considered by various models which postulate new unobserved, weakly-coupled light bosons in the MeV mass region [16-21]. A light boson could influence the charge form factor and its curvature at very low $Q^{2}$. The different behavior of the muon and electron could be achieved through fine tuning $[19,20]$ or with explicit breaking of lepton flavor universality [21]. In a constrained parameter space, such bosons could in principle be used to simultaneously explain the muon anomalous magnetic moment and to also provide a link to dark matter.

Whatever scenario turns out to be true, some of the implications can be studied with scattering experiments. If the puzzle is solely present in the bound-state physics of hydrogen, then muon and electron scattering should both give consistent values for the proton radius from form factor measurements. If new physics fundamentally distinguishes how muons and electrons interact with the proton, e.g. through a light boson that peferrably interacts with the muon, then a comparison of electron and muon scattering should reveal a difference in the extracted radii from both probes. If the proton polarizability effect is responsible, different sizes of two-photon exchange effects would be observable for $e^{ \pm} p$ and $\mu^{ \pm} p$ elastic scattering.

\section{The MUSE Experiment}

In order to shed light on this puzzle and its possible resolution, the MUSE collaboration has proposed an experiment at the Paul-Scherrer Institute (PSI) to verify the consistency of the proton radius extractions from elastic scattering with electronic and muonic probes of either charge in a simultaneous measurement [22]. MUSE has been approved by the PAC since the beginning of 2013. The idea is to use a common beam of $e$ and $\mu$ of either charge to perform elastic scattering from the proton at low momentum transfer. Comparisons of $e p$ and $\mu p$ elastic scattering will be made for the obtained yield, cross section, charge form factor, and extracted charge radius from the form factor slope with $Q^{2}$ for each of the four probes $e^{ \pm}, \mu^{ \pm}$. The possibility to reverse the charge sign of the beam allows to study any two-photon exchange effects, which are controversial [13-15]. The MUSE experiment directly tests the most interesting possible explanations of the proton radius puzzle, that there are differences in the $\mu p$ and $e p$ interactions. The MUSE setup is shown schematically in the left half of Fig. 1. 
PSI produces a mixed secondary beam of pion, muons and electrons. The $500 \mathrm{MeV}$ high-power proton cyclotron operates in pulsed mode at $50 \mathrm{MHz}$ bunch frequency, i.e. every $20 \mathrm{~ns}$ a short pulse of secondary particles is emitted from the production target. The $\pi \mathrm{M} 1$ channel selects momentum and charge of the particles and delivers the charged particle beam to the experiment through a double-C type magnetic chicane, where it is collimated at an intermediate dispersive point to limit the maximum intensity to $5 \mathrm{MHz}$. Quadrupole magnets are used to produce a beam focus of $1-2 \mathrm{~cm}$ in diameter at the target. The secondary beam is cleanly separated by measuring the time of flight between RF signal and arrival time using scintillating fiber hodoscopes. Measurement of the beam position in the dispersive plane between the dipole chicane magnets provides the momentum of the beam particle to better than $1 \%$ precision. For selected momenta of 115,153 , and $210 \mathrm{MeV} / \mathrm{c}$, the arrival times of pions, muons and electrons differ by at least $4 \mathrm{~ns}$ each, which allows rejecting pions at the trigger level. For each event in the experiment triggered by the scattered particle in combination with RF time and beam scintillating fiber hodoscopes, the beam particle species will be known very cleanly. At the center of the experiment is a liquid hydrogen target. The length of the target cell will be $4 \mathrm{~cm}$, optimized to balance the expected yield with the tolerable resolution due to multiple scattering. The main detector for the scattered particles will be based on wire chambers for tracking and fast time-offlight scintillator walls for trigger, timing and particle identification. Simulations have shown that the time-of-flight technique is sufficient at the low momenta for all purposes and Cerenkov detectors are not needed for particle identification. While the time resolution from the beam hodoscopes is at the $\mathrm{ns}$ level, the scintillators of the main detector have a time resolution of $50 \mathrm{ps}$. The addition of a fast quartz Cerenkov counter in front of the target allows to measure the time of flight for the scattered particle to $100 \mathrm{ps}$. This identifies the scattered particle type and eliminates most of the in-flight decay background.

For the cross section measurements, kinematic information needs to be sufficiently precise and accurate. Since the beam has an envelope of the order of a few $\mathrm{cm}$ allowing for angle variations of
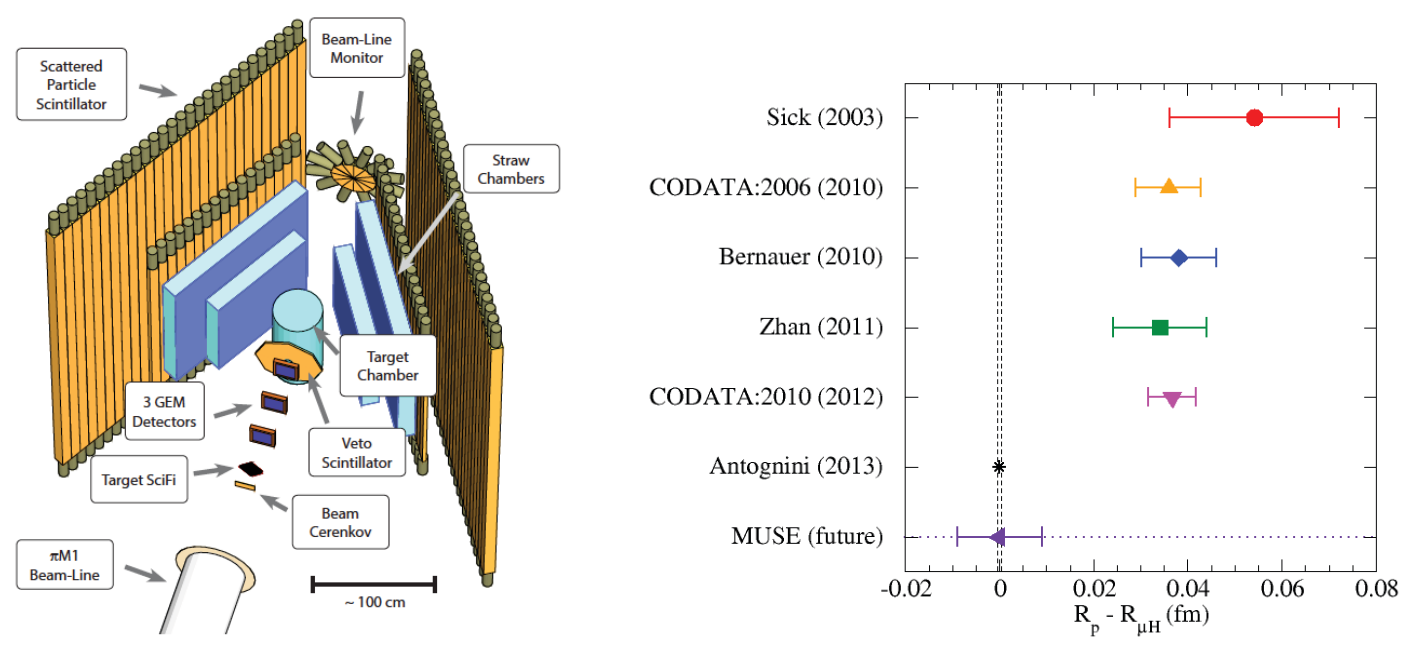

Figure 1. Left: Schematic view of the MUSE setup, consisting of a mixed, collimated e/ $\mu / \pi$ beam, scintillatingfiber hodoscopes for time-of-flight and position, GEM detectors for precise beam position, liquid hydrogen target, as well as drift tube chambers and scintillator walls to detect scattered particles. Right: Sensitivity of MUSE to the difference of proton charge radii between electron and muon probes, all relative to the Pohl data point [4]. 
the order of $100 \mathrm{mrad}$, a key instrument will be a beam tracker with high spatial resolution that can provide information of the angle of the incoming beam particle to better than $10 \mathrm{mrad}$, event-by-event. The beam tracker is exposed to the entire beam flux of $5 \mathrm{MHz}$ while the experiment trigger rate will be of the order of $2 \mathrm{kHz}$. Therefore, Gas Electron Multiplier detectors will be used, which have become available from the recently decommissioned OLYMPUS experiment at DESY [23]. Three GEM elements are arranged as a tracking telescope in front of the target region, providing a 3D beam tomography. Event-by-event, measurement of the scattering angle with the main detector will be in reference to the incoming track determined by the GEM beam telescope. The precision and accuracy of the MUSE experiment has been designed to verify the observed discrepancy in the charge radius, if confirmed, with about four standard deviations of significance. The right half of Fig. 1 shows the sensitivity of MUSE to the difference of proton charge radii between electron and muon probes, all relative to the first muonic hydrogen data point [4].

\section{Acknowledgements}

The author has been supported for this work by the National Science Foundation under grant PHY-1207672 and by DOE Early Career Award DE-SC0003884.

\section{References}

[1] R. Hofstadter, Rev. Mod. Phys. 28, 214 (1956).

[2] J.C. Bernauer et al., Phys. Rev. Lett. 105, 242001 (2010).

[3] X. Zhan et al., Phys. Lett. B 705, 59 (2011).

[4] R. Pohl et al. (CREMA coll.), Nature 466, 213 (2010).

[5] P.J. Mohr, B.N. Taylor and D.B. Newell, Rev. Mod. Phys. 80, 633 (2008).

[6] A. Antognini et al. (CREMA coll.), Science 339, 417 (2013).

[7] P.J. Mohr, B.N. Taylor and D.B. Newell, Rev. Mod. Phys. 84, 1527 (2012).

[8] R. Pohl, R. Gilman, G.A. Miller, and K. Pachucki, Annu. Rev. Nucl. Part. Sci. 63 (2013).

[9] K. Pachucki, Phys. Rev. A 60, 3593 (1999).

[10] Th. Walcher, arXiv:1207.4901v2, (2012).

[11] U.D. Jentschura, Ann. Phys. (N.Y.) 326, 500 (2011).

[12] E. Borie, Ann. Phys. 327, 733 (2012).

[13] C.E. Carlson and M. Vanderhaeghen, Phys. Rev. A 84, 020102 (2011).

[14] M.C. Birse and J.A. McGovern, Eur. Phys. J. A 48, 120 (2012).

[15] G.A. Miller, Phys. Lett. B 718, 1078 (2013).

[16] M. Pospelov, Phys. Rev. D 80, 095002 (2009).

[17] V. Barger, C.W. Chiang, W.Y. Keung, D. Marfatia, Phys. Rev. Lett. 106, 153001 (2011).

[18] D. Tucker-Smith and I. Yavin, Phys. Rev. D 83, 101702 (2011).

[19] C.E. Carlson and B.C. Rislow, Phys. Rev. D 86, 035013 (2012).

[20] C.E. Carlson and B.C. Rislow, Phys. Rev. D 89, 035003 (2013).

[21] B. Batell, D. McKeen, and M. Pospelov, Phys. Rev. Lett. 107, 011803 (2011).

[22] R. Gilman et al., Studying the Proton "Radius" Puzzle with $\mu$ p Elastic Scattering, White Paper on the Paul Scherrer Institut MUSE experiment, arXiv:1303.2160 (2013).

[23] R. Milner, D. Hasell, M. Kohl, U. Schneekloth et al., “The OLYMPUS Experiment”, Nucl. Instr. Meth. Phys. Res. A 741, 1 (2014). 